\title{
A case of poorly differentiated hepatocellular carcinoma with intriguing ultrasonography findings
}

\author{
NORITAKA WAKUI $^{1}$, RYUJI TAKAYAMA ${ }^{1}$, YASUSHI MATSUKIYO ${ }^{1}$, TAKANORI MUKOUZU $^{1}$, \\ MASAHIRO KANAYAMA ${ }^{1}$, MASAYOSHI TAKAHASHI ${ }^{1}$, HIDENARI NAGAI $^{1}$, MANABU WATANABE ${ }^{1}$, \\ KENICHI MARUYAMA ${ }^{2}$, MASAHIRO KANEKO ${ }^{3}$, KAZUTOSHI SHIBUYA ${ }^{4}$ and YASUKIYO SUMINO ${ }^{1}$ \\ Divisions of ${ }^{1}$ Gastroenterology and Hepatology, ${ }^{2}$ Clinical Functional Physiology, ${ }^{3}$ Hepato-Biliary-Pancreatic Surgery \\ and ${ }^{4}$ Surgical Pathology, Toho University Omori Medical Center, Ota-ku, Tokyo 143-8541, Japan
}

Received March 6, 2012; Accepted June 13, 2012

DOI: $10.3892 / 01.2012 .764$

\begin{abstract}
A 60-year-old female was referred to the Toho University Omori Medical Center due to ultrasonography findings revealing a notable hepatic mass. The $44 \times 32 \mathrm{~mm}$ isoechoic mass had a high-echo band along the margin in the liver. Contrast-enhanced ultrasonography (CEUS) with Sonazoid detected an enhancement pattern extending from the outer periphery to the inside of the mass in the vascular phase and a pattern similar to that of surrounding hepatic tissue in the post-vascular phase. High-flow hepatic hemangioma was suspected due to the hyperechoic rim-like high-echo band, the oval shape and the CEUS findings. However, computed tomography revealed a hypervascular hepatocellular carcinoma (HCC) pattern and the patient underwent surgery. Histopathological findings revealed poorly differentiated HCC. As poorly and moderately differentiated HCC types are conventionally classified as 'hypervascular HCC', few detailed ultrasonography (US) studies of poorly differentiated HCC are available. US characteristics of hypervascular HCC include the presence of a halo (hypoechoic band) around the lesion and reduced signal intensity in the post-vascular phase of CEUS. US in the patient revealed a mass with a hyperechoic band and a signal intensity in the post-vascular phase of CEUS that was almost identical to that of the surrounding liver parenchyma. These findings suggest poorly differentiated $\mathrm{HCC}$ and indicate that further research on hypervascular $\mathrm{HCC}$ is required.
\end{abstract}

Correspondence to: Dr Noritaka Wakui, Division of Gastroenterology and Hepatology, Toho University Omori Medical Center, 6-11-1 Omorinishi, Ota-ku, Tokyo 143-8541, Japan

E-mail: noriwakui@yahoo.co.jp

Abbreviations: US, ultrasonography; CEUS, contrast-enhanced ultrasonography

Key words: poorly differentiated hepatocellular carcinoma, Sonazoid, contrast-enhanced ultrasonography, liver

\section{Introduction}

Similar to moderately differentiated hypervascular hepatocellular carcinoma (HCC), poorly differentiated HCC is generally rich in vasculature. Accordingly, these types of HCCs have often been classified together as "hypervascular HCC' and as a consequence, ultrasonography (US) studies focusing exclusively on poorly differentiated HCC are rare. Here, we study a case of poorly differentiated HCC for which we conducted a detailed analysis of US images. The study was performed with the approval of the ethics committee at Toho University Omori Medical Center and with the consent of the patient.

\section{Case report}

A 60-year-old female patient was diagnosed as being a hepatitis B virus (HBV) carrier at approximately 30 years of age. Routine annual physical examinations since the initial diagnosis detected no abnormality until 2007 when US findings indicated the presence of a hepatic mass in the S5 region. The patient was referred to the Toho University Omori Medical Center (Tokyo, Japan) for a complete physical examination in November 2007. No subjective symptoms were observed. The patient had no history of alcohol drinking or smoking; however, the mother of the patient had succumbed to hepatic cirrhosis B. Additionally, with the exception of hospitalization due to a gastric ulcer at the age of 20 years, the patient had no previous history of major illness. Physical examination findings at presentation included height $(151 \mathrm{~cm})$, weight $(45 \mathrm{~kg})$, blood pressure $(124 / 80 \mathrm{mmHg})$ and pulse rate $(60 \mathrm{bpm})$. In addition, no anemia, jaundice, leg edema, palpable superficial lymph nodes, heart murmurs or pulmonary rales on auscultation were detected. The patient's abdomen was soft and flat with no tenderness or splenomegaly, and neurological examinations revealed no abnormal findings.

Hematological findings revealed that the patient was positive for hepatitis B surface antigen (s) and e-antibody (e), but not for hepatitis $\mathrm{C}$ virus ( $\mathrm{HCV}$ ) antibody, and the HBV-DNA concentration was $5.2 \mathrm{LGE} / \mathrm{ml}$. The levels of 
Table I. Laboratory data of patient on admission.

\begin{tabular}{|c|c|}
\hline Hematological findings & Result \\
\hline \multicolumn{2}{|l|}{ Peripheral blood } \\
\hline $\mathrm{Hb}(\mathrm{g} / \mathrm{dl})$ & 13.1 \\
\hline $\mathrm{Ht}(\%)$ & 39.8 \\
\hline $\mathrm{RBC}(/ \mu \mathrm{l})$ & $407 \times 10^{4}$ \\
\hline $\mathrm{WBC}(/ \mu 1)$ & 5500 \\
\hline Plt $(/ \mu 1)$ & $24.5 \times 10^{4}$ \\
\hline \multicolumn{2}{|l|}{ Coagulation test } \\
\hline $\mathrm{PT}(\%)$ & 100.0 \\
\hline \multicolumn{2}{|l|}{ Blood chemistry } \\
\hline BUN (mg/dl) & 14 \\
\hline $\mathrm{Cr}(\mathrm{mg} / \mathrm{dl})$ & 0.57 \\
\hline $\mathrm{TP}(\mathrm{g} / \mathrm{dl})$ & 7.4 \\
\hline Alb (g/dl) & 4.5 \\
\hline AST (IU/l) & 49 \\
\hline ALT (IU/l) & 43 \\
\hline LDH (IU/l) & 385 \\
\hline $\operatorname{ALP}(\mathrm{IU} / \mathrm{l})$ & 240 \\
\hline$\gamma$-GTP (IU/l) & 11 \\
\hline T-bil (mg/dl) & 0.8 \\
\hline ChE (IU/l) & 330 \\
\hline AMY (IU/l) & 104 \\
\hline FBS (mg/dl) & 74 \\
\hline HbA1c (\%) & 5.1 \\
\hline CRP (mg/dl) & 0.2 \\
\hline \multicolumn{2}{|l|}{ Virus markers } \\
\hline HBsAg (expression) & + \\
\hline HBsAb (expression) & - \\
\hline HBeAg (expression) & - \\
\hline HBeAb (expression) & + \\
\hline HBV-DNA (LGE/ml) & 5.2 \\
\hline HCV-Ab (expression) & - \\
\hline \multicolumn{2}{|l|}{ Tumor markers } \\
\hline $\operatorname{AFP}(\mathrm{mg} / \mathrm{ml})$ & 2.7 \\
\hline PIVKA II (U/ml) & 11 \\
\hline CEA $(\mathrm{U} / \mathrm{ml})$ & 2.9 \\
\hline
\end{tabular}

$\mathrm{Hb}$, hemoglobin; Ht, hematocrit; RBC, red blood cells; WBC, white blood cells; Plt, platelets; PT, prothrombin; BUN, blood urea nitrogen; $\mathrm{Cr}$, creatinine ratio; TP, total protein; Alb, albumin; AST, aspartate aminotransferase; ALT, alanine aminotransferase; LDH, lactate dehydrogenase; ALP, alkaline phosphatase; $\gamma$-GTP, $\gamma$-glutamyl transpeptidase; T-bil, total bilirubin; ChE, cholinesterase; AMY, amylase; FBS, fasting blood sugar; HbA1c, hemoglobin A1c; CRP, C-reactive protein; HB, hepatitis B; s, surface; Ag, antigen; $\mathrm{Ab}$, antibody; e, e-antigen; HBV, hepatitis B virus; $\mathrm{HCV}$, hepatitis $\mathrm{C}$ virus; $\mathrm{AFP}, \alpha$-fetoprotein; PIVKA II, protein induced by vitamin $\mathrm{K}$ absence or antagonist II; CEA, carcinoembryonic antigen.

$\alpha$-fetoprotein (AFP), protein induced by vitamin $\mathrm{K}$ absence or antagonist (PIVKA) II and carcinoembryonic antigen (CEA) tumor markers were all within the normal range

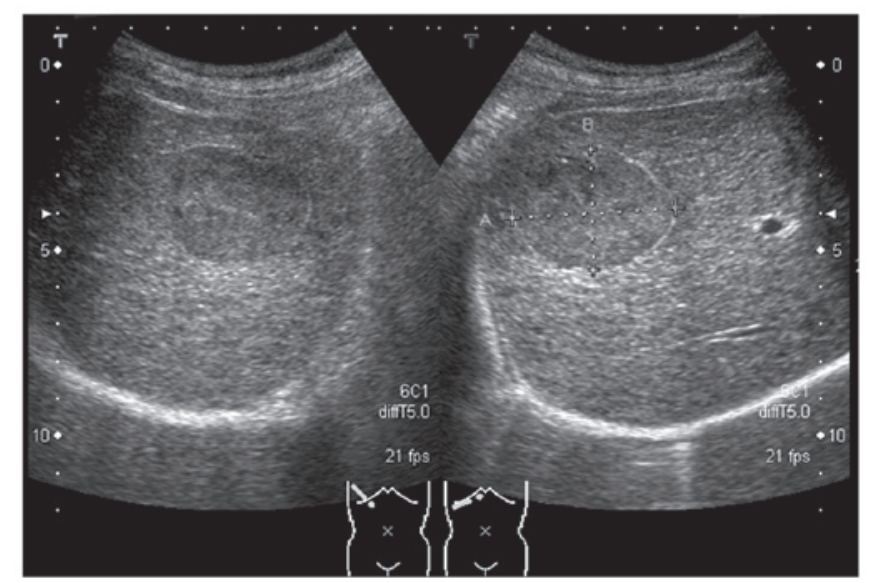

Figure 1. An abdominal ultrasonogram obtained at initial presentation in November 2007 demonstrating a 44x32 mm oval-shaped mass with a hyperechoic rim-like high-echo band along its margin, and an internal echo pattern homogeneous and almost isoechoic to the surrounding hepatic tissue in the S5 region of the liver.

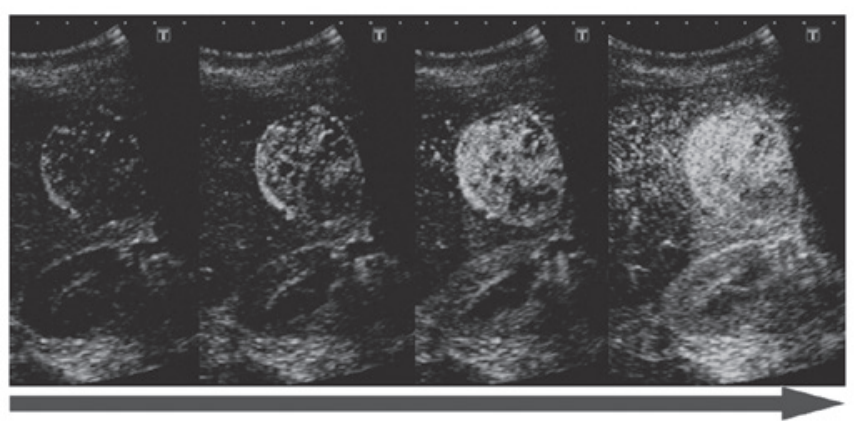

Figure 2. A Sonazoid-enhanced ultrasonogram obtained at initial presentation in November 2007 demonstrating an early enhancement pattern extending from the outside to the inside of the mass in the vascular phase.

(Table I). B-mode US revealed the presence of an ovalshaped mass $(44 \times 32 \mathrm{~mm}$ in diameter) in the S5 region of the liver. The mass had a hyperechoic rim-like high-echo band along the margin and an internal echo pattern, which was homogeneous and isoechoic to the surrounding hepatic tissue (Fig. 1). Contrast-enhanced US (CEUS) with Sonazoid demonstrated an early enhancement pattern extending from the outside to the inside of the mass in the vascular phase, and an enhancement pattern similar to that of the surrounding hepatic tissue in the post-vascular phase (Figs. 2 and 3). B-mode findings suggested that the lesion was a hemangioma, and due to the early enhancement pattern observed in the vascular phase of CEUS, the lesion was suspected to be a high-flow hemangioma. As the patient was a HBV carrier, we conducted magnetic resonance imaging (MRI) and computed tomography (CT) to eliminate the possibility of HCC. The mass was detected as low- and high-intensity signals on T1- and T2-weighted MRI images, respectively (Fig. 4). An abdominal CT scan revealed a low-density lesion in the plain phase, a heterogeneous high-density lesion in the early phase and a low-density lesion in the equilibrium 


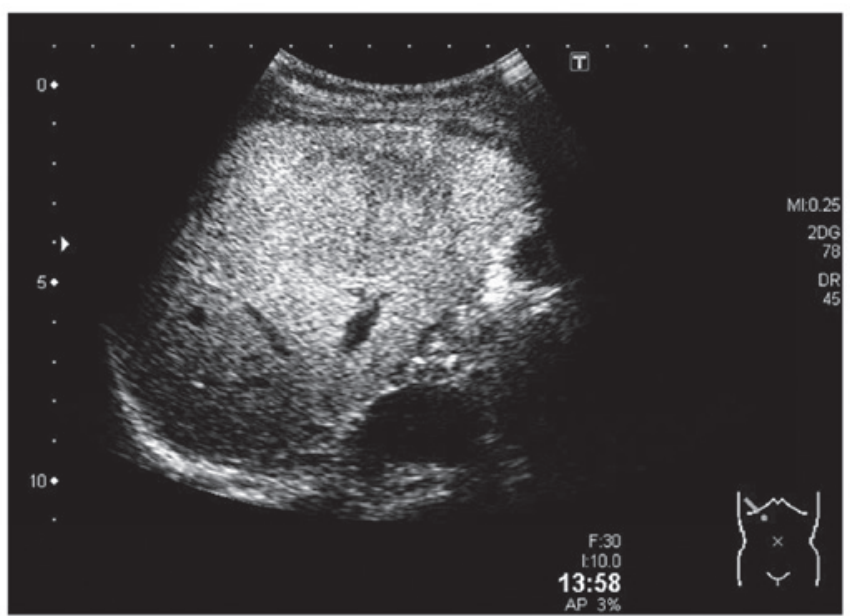

Figure 3. A Sonazoid-enhanced ultrasonogram obtained at initial presentation in November 2007 demonstrating an enhancement pattern similar to that of the surrounding hepatic tissue in the post-vascular phase.

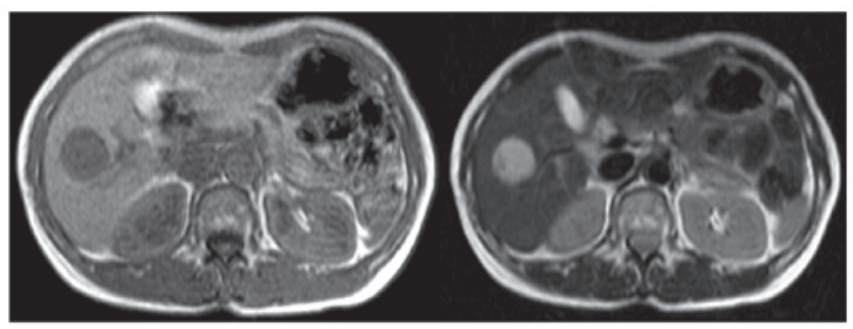

$\mathrm{T} 1-\mathrm{W}$

T2-W

Figure 4. Abdominal MRI obtained at initial presentation in November 2007 demonstrating a mass with low signal intensity on T1-W images and markedly high signal intensity, compared to the gall bladder, on $\mathrm{T} 2-\mathrm{W}$ images. T1-W, T1-weighted; T2-W, T2-weighted; MRI, magnetic resonance imaging.

phase (Fig. 5). Based on the collected results, the patient was diagnosed with hypervascular HCC and was subjected to laparoscopic right hepatic lobectomy.

Macroscopic findings revealed a brownish colour and an irregular surface of the liver (Fig. 6). No enlargement or atrophy was observed and the liver edge was reasonably sharp. Additionally, the tumor was a 40x28 mm grayish-white solid mass with a relatively clear margin and a capsule-like structure.

Histopathological findings identified solid tumor cells in the mass region (Figs. 7 and 8). Slit-like, or in certain parts, blood sinus-like spaces composed of endothelial cells were observed to be arranged in slightly ambiguous five-to-seven-layer cords. Tumor cells were not markedly eosinophilic and had an increased nuclear/cytoplasmic (N/C) ratio. Additionally, the cells had polymorphic and chromatin-rich nuclei. The mass was surrounded by a fibrous capsule, which was internally lined with a layer of lipid droplets as thick as the fibrous capsule, spreading along almost the entire circumference of the tumor. The pathological diagnosis was poorly differentiated HCC. The non-cancerous area was diagnosed as chronic hepatitis (F2/A1).
A

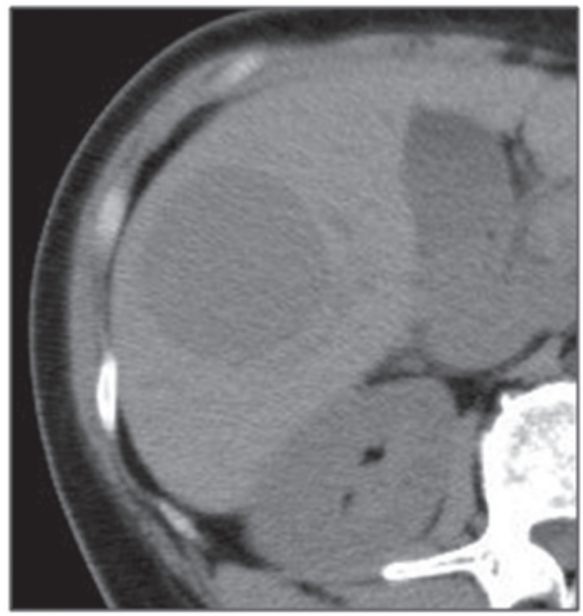

B

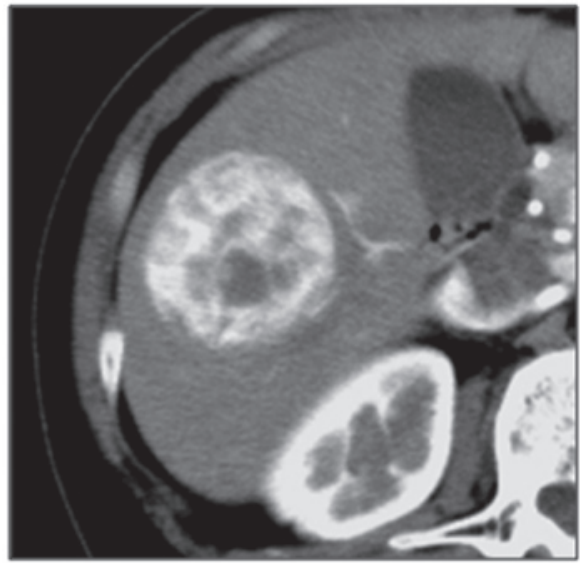

C

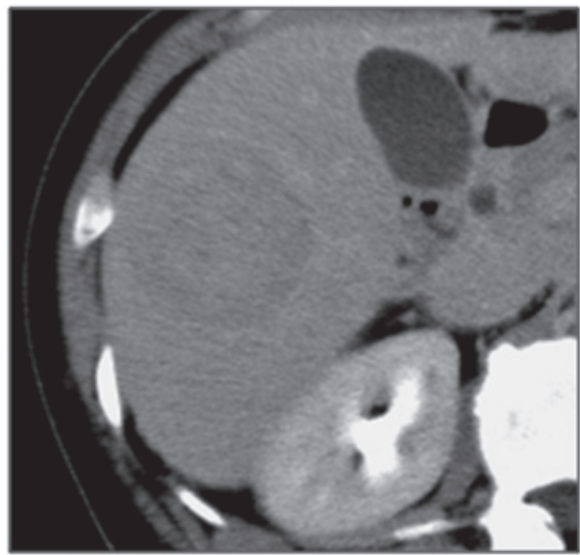

Figure 5. Contrast-enhanced abdominal CT images obtained in November 2007 demonstrating (A) a low-density mass in the plain phase, (B) a heterogeneous high-density mass in the early phase and (C) a low-density mass in the late phase. CT, computed tomography.

\section{Discussion}

Poorly differentiated $\mathrm{HCC}$ has a worse prognosis compared to well- and moderately differentiated HCC (1). Poorly differentiated $\mathrm{HCC}$ is also reported to have poor outcomes following living donor liver transplantation, which has increased in recent years (2). However, as poorly and moderately differentiated HCC have been grouped together as 'hypervascular 


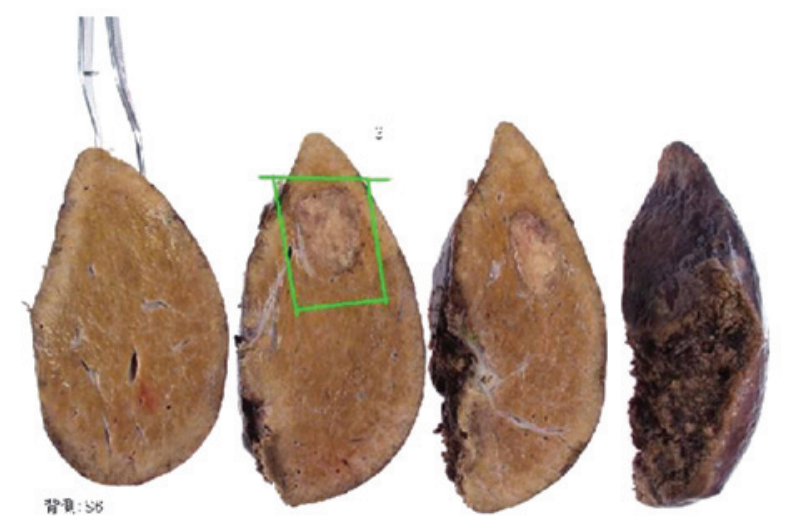

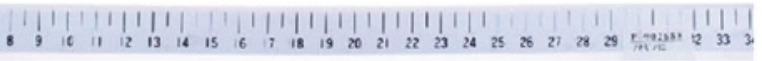

Figure 6. Macroscopic findings of the tumor. The liver appeared brownish and had an irregular surface. No enlargement or atrophy was observed and the liver edge was reasonably sharp. The tumor was a 30x20 mm grayish-white solid mass with a relatively clear margin and a capsule-like structure.

HCC', the imaging characteristics of the former have rarely been studied in detail.

According to previous studies, hypervascular HCCs with a diameter greater than $15 \mathrm{~mm}$ observed using US are often associated with a hypoechoic halo (3), and a CEUS with Sonazoid signal that is weaker than that in the surrounding liver during the post-vascular phase (4). However, in the present case, we observed imaging features that are uncharacteristic of hypervascular HCC, including a hyperechoic (not hypoechoic) band around the mass on B-mode US, and the absence of perfusion defects on CEUS with Sonazoid images in the post-vascular phase.

Possible reasons for the atypical US finding of a hyperechoic band are that, from the histological standpoint, the tumor was not only enclosed by a fibrous outer capsule, but also covered by a layer of lipid droplets of a width similar to that of the capsule. This lipid droplet layer is suggested to be the hyperechoic rim observed on B-mode US. It is unclear why the tumor was covered with a layer of lipid droplets; however, it is possible that these droplets were pushed outwards in the late maturation phase as the biological malignancy of the tumor increased.

The absence of perfusion defects on CEUS with Sonazoid images in the post-vascular phase may have been caused by portal vein tumor thrombus in the vicinity of the tumor. The number of Kupffer cells reduces as the biological malignancy of HCC progresses (5-7). Furthermore, the post-vascular phase of Sonazoid-enhanced US imaging mainly reflects the number and functional role of the Kupffer cells in the liver, indicating that, in the case of hypervascular HCC, defects are present during the post-vascular phase of US (4). However, in the present case, the tumor did not appear as a defect, but as an isoechoic mass compared to the surrounding liver in that phase. A reason for this may be the presence of a portal vein tumor thrombus, which appears progressively as HCC develops (8). If a tumor thrombus is present in the portal vein, which serves as the exit for tumor blood flow, the contrast

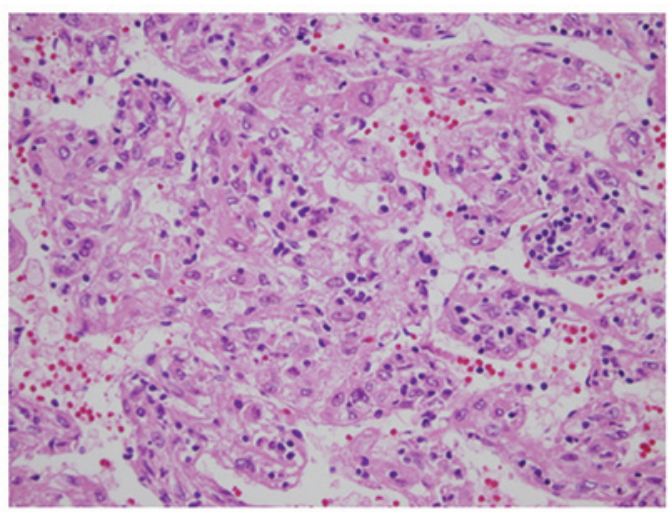

Figure 7. Histopathological findings of the tumor (H\&E staining). Solid tumor cells were observed in the mass region. Endothelial cells were arranged in slightly ambiguous five-to-seven-layer cords. Tumor cells were not strongly eosinophilic and had an increased N/C ratio. These cells had polymorphic and chromatin-rich nuclei. Magnification x400. H\&E, hematoxylin and eosin; N, nuclear; C, cytoplasmic.

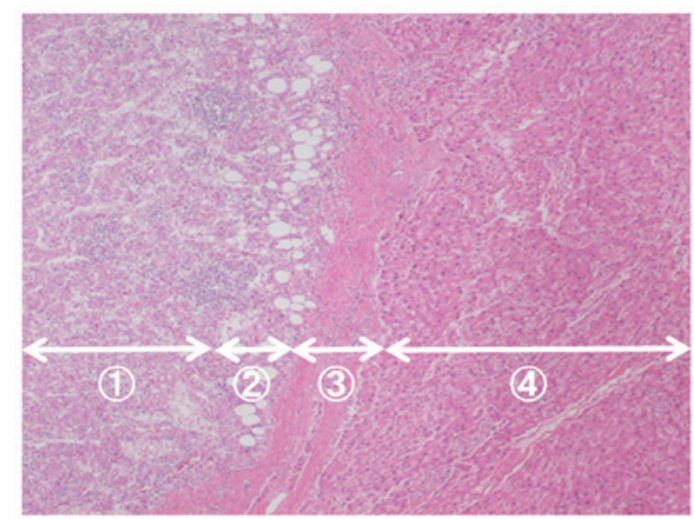

Figure 8. Histopathological findings of the tumor and non-tumor regions (H\&E staining). 1, Tumor region; 2, lipid droplets; 3, fibrous capsule; 4 , non-tumor region. The mass was surrounded by a fibrous capsule, which was internally lined with a layer of lipid droplets as thick as the fibrous capsule, spreading along almost the entire circumference of the tumor. Magnification x100. H\&E, hematoxylin and eosin.

agent that enters the tumor will be prevented from exiting the tumor. This would force the contrast agent to remain in the tumor and not cause a perfusion defect. According to the 17th Annual Survey and Follow-up Study of Primary Liver Cancer conducted by the Liver Cancer Study Group of Japan, poorly differentiated HCC has a higher incidence of portal vein tumor thrombus compared to moderately differentiated HCC (9). The study also revealed that poorly differentiated HCC had fewer defects on post-vascular phase Sonazoid-enhanced US images compared to moderately differentiated HCC.

Poorly differentiated HCC has a worse prognosis than well- and moderately differentiated HCC. Clinically, it is extremely important to make a precise diagnosis of the disease using diagnostic imaging techniques, particularly non-invasive US. Here, we studied an notable case of poorly differentiated HCC which offers an insight into the relatively unknown imaging characteristics of poorly differentiated HCC. 


\section{References}

1. Nakajima Y, Shimamura T, Kamiyama T, Kimura J, Sato N Matsushita M, Une Y and Uchino J: Evaluation of surgical resection for small hepatocellular carcinomas. Am J Surg 171: 360-363, 1996.

2. Jonas S, Bechstein WO, Steinmüller T, Herrmann M, Radke C, Berg T, Settmacher U and Neuhaus P: Vascular invasion and histopathologic grading determine outcome after liver transplantation for hepatocellular carcinoma in cirrhosis. Hepatology 33: 1080-1086, 2001.

3. Ohkuma K: Ultrasound diagnosis. Jpn J Cancer Clin 47: 987-994, 2001 (In Japanese).

4. Shunichi S, Hiroko I, Fuminori M and Waki H: Definition of contrast enhancement phases of the liver using a perfluoro-based microbubble agent, perflubutane microbubbles. Ultrasound Med Biol 35: 1819-1827, 2009.

5. Tobe K, Tsuchiya $\mathrm{T}$ and Fujiwara R: Kupffer cells in welldifferentiated tissue of hepatocellular carcinoma. Acta Hepatol Jpn 26: 630-637, 1985 (In Japanese with English abstract).
6. Sugihara S, Nakashima O and Kiyomatsu K: Pathomorphologic study on macrophages in hepatocellular carcinoma. Acta Hepatol Jpn 31: 12-18, 1999 (In Japanese with English abstract).

7. Tanaka M, Nakashima O, Wada Y, Kage M and Kojiro M: Pathomorphological study of Kupffer cells in hepatocellular carcinoma and hyperplastic nodular lesions in the liver. Hepatology 24: 807-812, 1996.

8. Yabuuchi I, Matuda Y and Ifuji T: Classification of contrast enhanced US images of small hepatocellular carcinoma using Levovist. Kan Tan Sui 47: 175-182, 2003 (In Japanese with English abstract).

9. Ikai I, Arii S, Okazaki M, Okita K, Omata M, Kojiro M, Takayasu K, Nakanuma Y, Makuuchi M, Matsuyama Y, Monden $M$ and Kudo M: Report of the 17th Nationwide Follow-up Survey of Primary Liver Cancer in Japan. Hepatol Res 37: 676-691, 2007. 